\title{
Miniaturization of Waveguides Dual Band Antenna Using TSRR-WS Metamatrerials
}

\author{
M. Jalali, T. Sedghi, Y. Zehforoosh, Department of Electrical Engineering, IAU, Urmia, Iran
}

\begin{abstract}
In this paper, an open - ended rectangular waveguide antenna loaded with Triangular Split Ring Resonators and Wire Strip (TSRR-WS) radiating below the cut-off frequency of the waveguide is proposed. This work has investigated design development, simulation and miniaturization of an open end a waveguide dual band antenna. A novel technique was employed to miniaturize the open-ended radiator using electromagnetic metamaterials (MTM). The antenna is capable of radiating below the cutoff frequency of the waveguide by supporting backward waves. Comparing previous work of miniaturization of waveguides, a better bandwidth about $600 \mathrm{MHz}$, good matching and low profile (with 5 unit cell of MTMs) antenna is obtained.
\end{abstract}

Index Terms—dual band antenna, metamaterials, waveguide

\section{INTRODUCTION}

In the last few years, there have been several new ideas which may to the miniaturization of waveguides [1]. Recently, a very unusual waveguide was proposed by Marques et al. in [2] and then extensively studied by Hrabar et al. in [3]. Marques et al. proposed a rectangular metallic waveguide periodically loaded with resonant magnetic scatterers, so-called split - ring resonators (SRR's) [3]-[6], [9], [10]. The design of split rings is very important to construct a new type of MTMs. Numerous types of different ring and ring-like structures such as circular, square, $\Omega$-shaped, U-shaped, S-shaped and others are used to create new MTMs [7]. In the light of the known structures we decided to use a new MTM which consist of a triangular shaped ring which has been proposed in[8]. We use this structure in an open ended waveguide antenna and do simulation in the frequency range of 5-9 GHz. Simulation results are presented below. This kind of resonator has negative permittivity and permeability in the used frequency band. An X-band, open ended waveguide antenna loaded with five unit cells TSRR-WS, which is radiating below the cutoff frequency with a good bandwidth and better impedance matching, is designed and simulated.

Manuscript received May 10, 2009. This work was supported in part by the Islamic Azad university of Urmia branch.

M. Jalali is with the Islamic Azad University of Urmia branch, Iran (phone: 098-914-389-9112); e-mail: Jalali.mahdi@gmail.com).

T. Sedghi was with the Islamic Azad University of Urmia branch, Iran. He is now with the Department of electrical engineering, Urmia University, Iran (e-mail: st t.sedghi@urmia.ac.ir).

Y. Zehforoosh is with the Electrical Engineering Department, Islamic Azad university of Urmia, Iran, (e-mail: y.zehforoosh@ srbiau.ac.ir).

\section{II.THEORETICAL ANALYSIS AND SIMULATION}

In the simulations first we use combination of $\mathrm{SRR}^{2}$ and $\mathrm{WS}^{3}$ structures to fabricate artificial MTM. Figure 1 illustrates the geometry of the unit cell comprised of TSRR and WS.

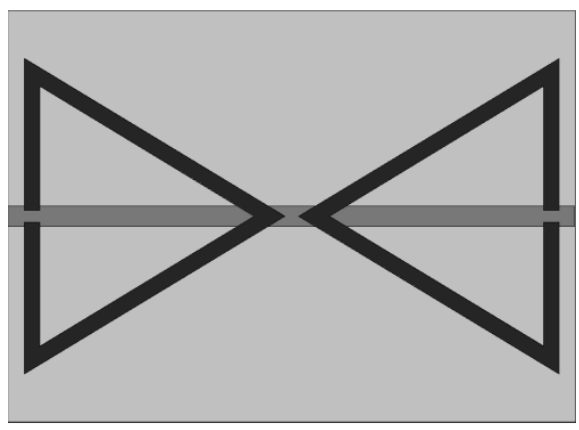

Figure 1. Unit cell of new metamaterial

A FR4 sheet of $0.25 \mathrm{~mm}$ thickness (with relative permittivity 4.4) is used as substrate for each configuration. TSRR and WS are made of copper with conductivity of $5.8 \times 10^{7} \mathrm{~S} / \mathrm{m}$. the width of all TSRRs is $0.4 \mathrm{~mm}$. TSRRs are located on one face of FR4 and WSs are etched on their opposite face. We use Ansoft's High Frequency Structure Simulator (HFSS) software; based on finite-element method (FEM).The width of WS is chosen $0.5 \mathrm{~mm}$. The base and height of TSRR are 7.794 and $6.75 \mathrm{~mm}$ respectively. The gap in each TSRR is $0.3 \mathrm{~mm}$ and space between TSRRs is $0.4 \mathrm{~mm}$ [8].

Left handed medium is simulated by a regular array of five TSRR-WS placed in the symmetry plane of $60 \mathrm{~mm}$ long. In a lossless case, the longitudinal propagation factor of this waveguide is given by the simple set of equations [4]:

$$
k_{z}= \pm k_{0} \sqrt{\varepsilon_{r} \mu_{t r}\left[1-\left(\frac{f_{c}}{f}\right)^{2}\right]}, f_{c}=\frac{f_{c 0}}{+\sqrt{\varepsilon_{r} \mu_{l r}}}, f_{c 0}=\frac{m c}{2 a} \quad m=1,2,3, \cdots
$$

Here, $k_{z}$ stands for waveguide propagation factor, $k_{\mathrm{o}}$ is a free space propagation factor, $\varepsilon_{r}$ is relative permittivity and $\mu_{t r}$ and $\mu_{l r}$ stand for relative permeability in transversal(x) and longitudinal (z) directions of the waveguide, respectively. The symbol $f$ stands for frequency of the signal, whereas $f_{c o}$ and $f_{c}$ are the cut-off frequency of an empty waveguide and the waveguide filled with a material respectively. The $\mathrm{X}$-band waveguide loaded with TSRR-WS is excited by a 
C-band waveguide-to-coaxial transition (cutoff frequency 4.3 $\mathrm{GHz}$ ). To ensure the excitation of the first SRR in the array, the first ring was partially placed out from the X-band waveguide; and to improve the radiation, the last ring was partially placed out from the open end of the waveguide along with an extra ring as shown in figure 2.

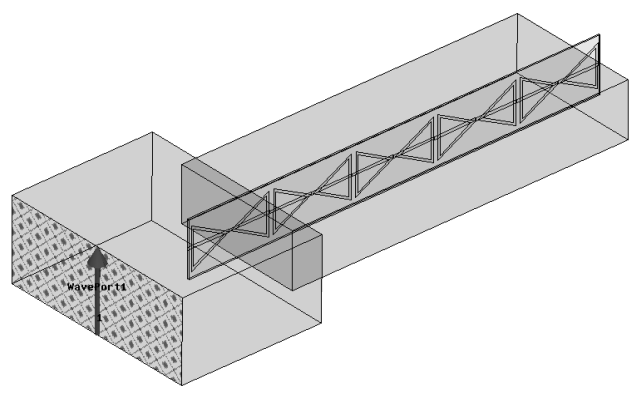

Figure 2. Schematic of the waveguide antenna with 5 unit cell of MTMs

Reflection coefficient $S_{11}$ at the input port for antenna is plotted in figure 3 . From the plot, one can notice that the propagation passband is located well below the cut of frequency of X-band waveguide with the bandwidth 600 $\mathrm{MHz}$. Minimum return loss computed is $18 \mathrm{~dB}$. Comparing this to previous works of miniaturization of waveguides in [2], [3], a larger bandwidth obtained with low profile and the less number of cells.

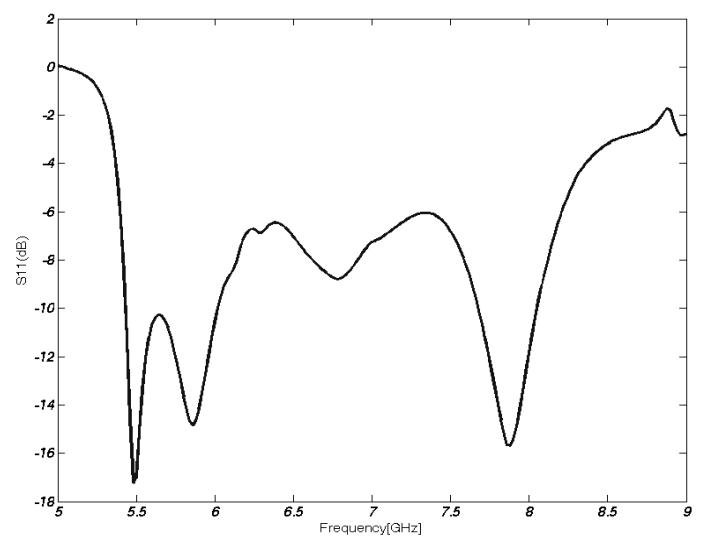

Figure 3. Return loss plot for Antenna with 5 unit cells

Obtaining passband below the cutoff frequency of the waveguide is not proof of a backward wave. In figure 4, which shows the phase and magnitude of the guided wave, it is clearly seen that in the passband, the phase of the wave increases unlike as in an ordinary waveguides where phase decreases. Thus, physically longer waveguides exhibit larger phase of $S_{11}$. This is because the direction of the phase velocity is opposite to the energy flow. This proves that there is a phase advance in such waveguides unlike phase delay in an ordinary waveguide. With this, one can conclude, physically longer backward wave waveguide appears electrically shorter with phase advance (figure 4).

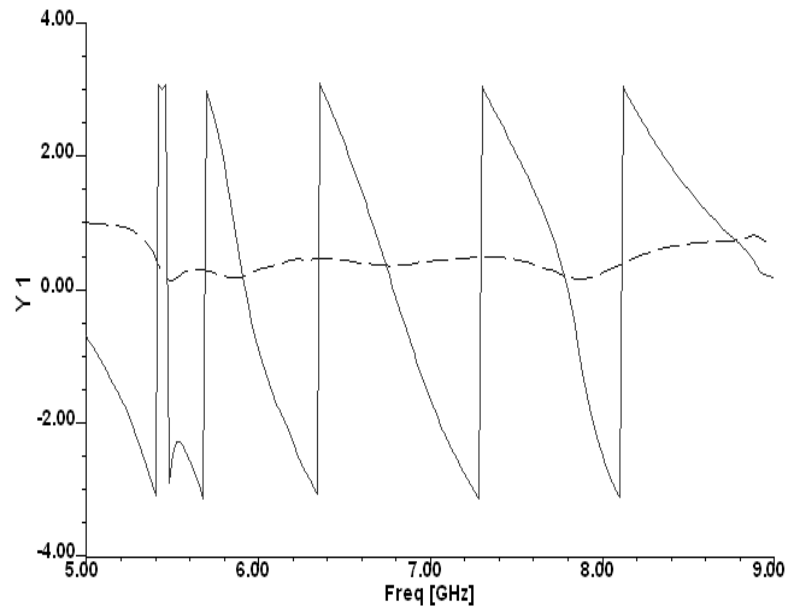

Figure 4. Phase (solid) and magnitude (dotted) of S11 for antenna with 5 unit cells

In the figure 5 and 6 E-plane and H-plane patterns of the antenna in resonance frequencies are depicted. The patterns show that the antenna works like an omini directional antenna on resonance frequencies.

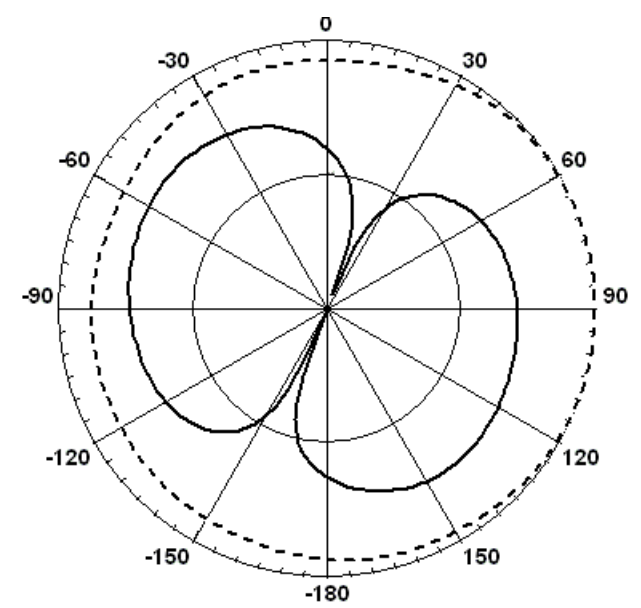

Figure 5. E-plane (solid) and H-plane (dotted) of antenna with 5 unit cells in $5.48 \mathrm{GHz}$

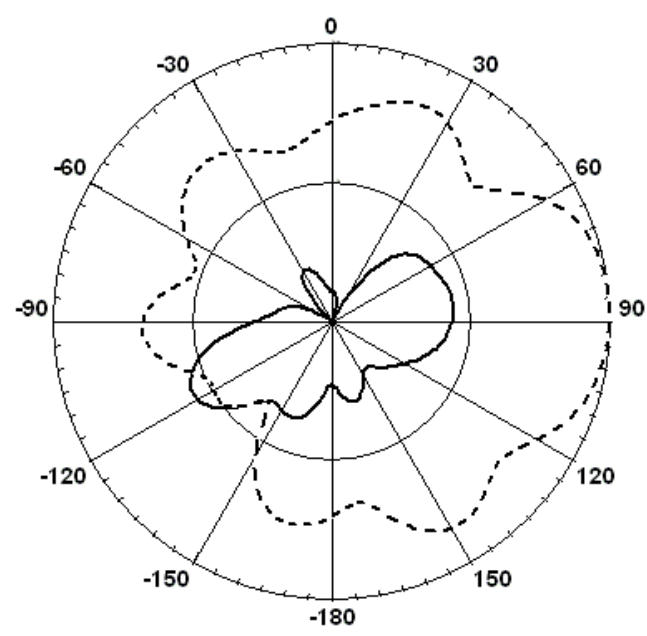

Figure 6. E-plane (solid) and H-plane (dotted) of antenna with 5 unit cells in $7.8 \mathrm{GHz}$

\section{PARAMETRIC ANALYSIS}

There are lots of parameters that affect on the antenna characteristics such as return loss and bandwidth. To see the effect of the parameters, there are some parametric analyses 
below.

\section{A. Analysis of waveguide antenna for different number of cells}

For analysis of the antenna characteristics for different number of cells we use 3, 4, 5 and 6 cells in the antenna and do simulation. The $S_{11}$ is presented in figure 7. results shows that the bandwidth is fully depend on the number of cells used in the antenna. Also it is obvious that a larger band width with the respect of cut-off frequency of the waveguide is obtained when we use 5 cells in the antenna.

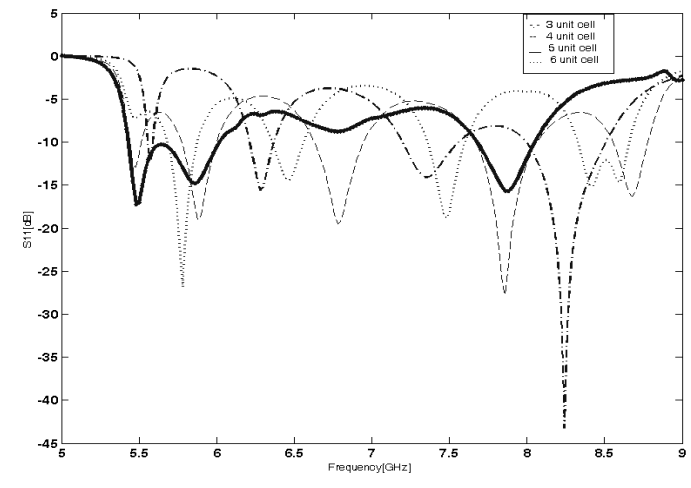

Figure 7. Return loss plot for Antenna with different number of cells

\section{B. Analysis of waveguide antenna for different dielectric substrate}

To see the effect of using different dielectric substrates in the waveguide antenna structure we use a Rogers RT/duroid 5870 with relative permittivity of 4.4 and dielectric loss tangent of 0.0012. Simulation results are presented in figure 8 As it is obvious using a substrate with lower relative permittivity cause a shift in bandwidth frequency range for lower band and a smaller bandwidth for higher band. So choosing FR4 as a proper dielectric substrate to have a lager bandwidth and less return loss is suitable.

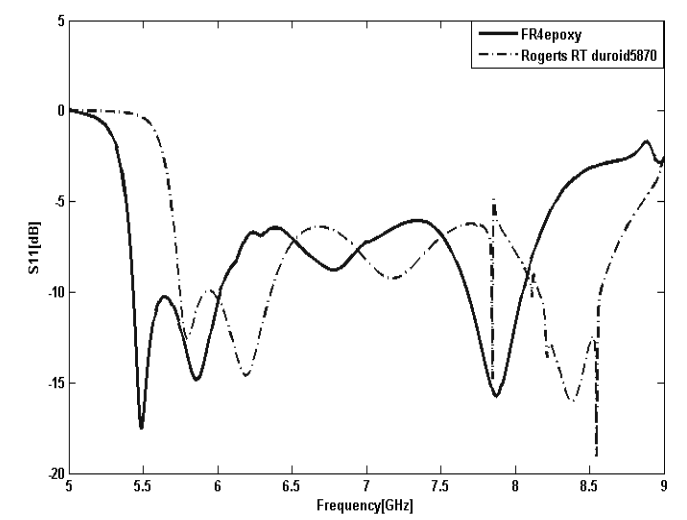

Figure 8. Return loss plot for Antenna with different substrates

\section{CONCLUSION}

Simulation of TSRR-WS loaded X-band waveguide antenna radiating below the cutoff frequency was successfully carried out. According to simulations, the antenna operated about $5 \mathrm{GHz}, 1 \mathrm{GHz}$ below the cut off frequency of the waveguide. The antenna had a bandwidth of $600 \mathrm{MHz}$. The primary goal of the research was to miniaturize a waveguide antenna using novel electromagnetic metamaterial rather than classical dielectric inclusion. The radiation property of the antenna does not depend on the cross section of the waveguide, but on the inclusion. Transverse width of such a waveguide can be, in principle, arbitrarily small. The lowest frequency of radiation, and therefore the miniaturization, is dictated by feasibility of fabrication of the metamaterial at the frequency of interest. We design a smaller, compact, and low profile antenna with better bandwidth from before works.

\section{ACKNOWLEDGMENT}

The authors would like to thank Islamic Azad university of uromia branch for it's support.

\section{REFERENCES}

[1] Waveguide handbook, Nathan Marcuvitz, 1986, IET

[2] R. Marques, J. Martel, F. Mesa, and F. Medina, "Left-handed-media simulation and transmission of EM waves in subwavelength split-ring-resonator-loaded metallic waveguides," Phys. Rev. Lett., pp. 183 901-183 904, Oct. 2002.

[3] Silvia Harbar, Juraj Bartolic, Zvonimir Sipus, "Waveguide Miniaturization Using Uniaxial Negative Permeability Metamaterial", IEEE TRANSACTIONS ON ANTENNAS AND PROPAGATION, VOL. 53, NO. 1, JANUARY 2005.

[4] X.-C. Zhang,Z.-Y. Yu,and J. Xu, "Novel band -pass substrate intenerated waveguide (SIW) filter based on complementary split ring resonators(CSRRS)" Progress In Electromagnetics Research, PIER 72, 39-46, 2007

[5] J.-X. Niu, X.-L. Zhou, andL.-S. Wu, "Analysis and application of novel structures based on split ring resonators structures based band on split ring resonators and coupled lines" Progress In Electromagnetics Research, PIER 75, 153-162, 2007

[6] S. Xi, H. Chen, B.-I. Wu and J. A. Kong, "Experimental confirmation of guidance properties using planar anisotropic left-handed metamaterials slabs based on s-ring resonators," Progress In Electromagnetics Research, PIER 84, 279-287, 2008

[7] J. B. Pendry, A. J. Holden, D. J. Robbins, and W. J. Stewart, "Magnetism from conductors and enhanced nonlinear phenomena," IEEE Trans. Microwave Theory Tech., vol. 47, p. 2075, 1999.

[8] [8] C.Sabah and S.Uckun"Traingular Spit Ring Resonator and Wire Strip to From New Metamatrial" URSI General Assembly Proceeding pp. 216- 220, August 2008.

[9] D. R. Smith, W. J. Padilla, D. C. Vier, S. C. Nemat-Nasser, and S. Schultz, "A composite medium with simultaneously negative permeability and permittivity," Phys. Rev. Lett., vol. 84, no. 18, pp. 4184-4187, May 2000.

[10] C. Caloz, A. Sanada, and T. Itoh, "Microwave circuits based on negative refractive index material structures," in Proc. EuMc 2003,Munich, 2003, pp. 389-392. X.-L. Zhou and L.-S. Wu, “Analysis and application of novel structures based on split ring resonators structures based band on split ring resonators and coupled lines" Progress In Electromagnetic Research, PIER 75, 153-162, 2007.

Mehdi Jalali was born in Urmia, Iran, in 1980. He received his B.S. degree in telecommunication engineering from Department of Electrical Engineering, Urmia Azad University in 2003 and M.S. degree from Department of Electrical Engineering, Tehran Azad University in 2006. Since 2006, he has taught telecommunication courses in Urmia Azad University. His research interests include electromagnetic fields.

Tohid Sedghi was born in Urmia, Iran, in 1986. He received his B.S. degree from Department of Electrical Engineering, Urmia Azad University in 2008. $\mathrm{He}$ is studying his M.S. degree at Department of Electrical Engineering Urmia University, Iran. His research interests include electromagnetic fields.

Yashar Zehforoosh was born in Urmia, Iran, in 1981. He received the B.S. degree in telecommunication engineering from Urmia Azad University, Urmia, Iran, in 2003, and the M.S. degree in wave and field engineering from the Urmia University, Iran, in 2007. He is currently working toward the Ph.D. degree in science and research branch of IAU University, Tehran, Iran. His research interests include microstrip antennas, UWB antennas and mobile small antennas. 